\title{
Molecular theory and the effects of solute attractive forces on hydrophobic interactions
}

\author{
Mangesh I. Chaudhar ${ }^{*}$ and Susan B. Remp£ \\ Center for Biological and Material Sciences, Sandia National Laboratories, Albuquerque, NM 87185 \\ D. Asthagir用 \\ Department of Chemical and Biomolecular Engineering, Rice University, Houston, TX 77005 \\ L. Tan\$ and L. R. Pratt \\ Department of Chemical and Biomolecular Engineering, Tulane University, New Orleans, LA 70118
}

(Dated: May 25, 2022)

\begin{abstract}
The role of solute attractive forces on hydrophobic interactions is studied by coordinated development of theory and simulation results for Ar atoms in water. We present a concise derivation of the local molecular field (LMF) theory for the effects of solute attractive forces on hydrophobic interactions, a derivation that clarifies the close relation of LMF theory to the EXP approximation applied to this problem long ago. The simulation results show that change from purely repulsive atomic solute interactions to include realistic attractive interactions diminishes the strength of hydrophobic bonds. For the Ar-Ar rdfs considered pointwise, the numerical results for the effects of solute attractive forces on hydrophobic interactions are of opposite sign and larger in magnitude than predicted by LMF theory. That comparison is discussed from the point of view of quasi-chemical theory, and it is suggested that the first reason for this difference is the incomplete evaluation within LMF theory of the hydration energy of the Ar pair. With a recent suggestion for the system-size extrapolation of the required correlation function integrals, the Ar-Ar rdfs permit evaluation of osmotic second virial coefficients $B_{2}$. Those $B_{2}$ also show that incorporation of attractive interactions leads to more positive (repulsive) values. With attractive interactions in play, $B_{2}$ can change from positive to negative values with increasing temperatures. This is consistent with the historical work of Watanabe, et al., that $B_{2} \approx 0$ for intermediate cases. In all cases here, $B_{2}$ becomes more attractive with increasing temperature.
\end{abstract}

\section{INTRODUCTION}

The concept of a hydrophobic interaction is firmly embedded in general views of the folding of water-soluble protein molecules. Kauzmann [1] clearly articulated that idea: "Thus, proteins are stabilized by the same physical forces as those that keep oil and water from mixing ..." A key phenomenological point is that enthalpic hydration contributions to the thermodynamics of protein unfolding decrease, even vanish at moderate temperatures [1]. Hydrophobic interactions are then entropy dominated. The enthalpy-entropy balance depends importantly on temperature, and switches at higher temperatures [1. The entropic hydration contributions to the thermodynamics of protein unfolding can vanish at higher temperatures [1, and that condition has been called the "entropy convergence" point 2. Nevertheless, below such an entropy convergence temperature, i.e., where hydrophobic low-solubility is associated a negative entropy change, hydrophobic interactions get stronger with temperature increases though with reduced rate of strengthening.

\footnotetext{
* michaud@sandia.gov

$\dagger$ slrempe@sandia.gov

$\ddagger$ Dilip.Asthagiri@rice.edu

$\S \overline{\text { Itan2@tulane.edu }}$

ฯ $\overline{\text { lpratt@tulane.edu }}$
}

From early days, the involvement of the hydration entropy has been conceptualized by imagining icebergs surrounding simple hydrophobic solutes, such as inert gases, e.g., Ar below. Tanford [3] attributed the original "iceberg" language to G. N. Lewis. Silverstein [4, decades later, provides a modern view of the relevance of the iceberg concept to hydrophobic phenomena. "Iceberg" is a widely recognized figure of speech, but has not been the basis of defensible statistical mechanical theory of these entropy effects. In fact, the statistical mechanical theory that eventually does explain the entropy convergence phenomena does not define or characterize iceberg structures [2].

Because the iceberg parlance is vague and provocative, direct experimental demonstrations of so-called inversetemperature behaviors are particularly helpful. Aggregation of sickle hemoglobin is a standard example 5 . Well-known aqueous polymers that separate with temperature increases, i.e., systems that exhibit a lower critical solution temperature (LCST), also provide examples. Elastin-like peptides (ELPs) are probably the best known cases [6 10]. Substantial molecular simulation work is available describing ELP collapse 11-18 without addressing the statistical mechanical theory of hydrophobic interactions. Those descriptive simulation efforts are largely consistent with the traditional idea of the folding of elastin-like peptides upon heating, and with each other, but not entirely [17. 
Aqueous solutions of poly(N-isopropylacrylamide)s provide other examples of LCSTs [19, 20. Polyethylene glycols (PEGs) in water also exhibit LCSTs 21. The polymers noted are water-soluble below their LCSTs. Thus they are substantially hydrophilic. But though they are complicated molecules, they directly demonstrate the inverse-temperature phenomena of classic hydrophobic effects.

The successful statistical mechanical theory for the entropy convergence behavior [22 29] developed over decades from counter-intuitive initial steps 30 38. The statistical mechanical theory of hydrophobic interactions 35, 39 was formulated for hard sphere hydrophobic solutes in water, and theoretical progress has been associated with attention to detail for such simple cases [24, 35, 39. That methodical analysis strategy permits clarity in isolating the features that are the ultimate interest. An important accomplishment of recent work 39] was then to prove numerically that rigorously defined hydrophobic interactions between atomicsized hard sphere solutes in water also exhibit inversetemperature behavior. Independently, new results for broader solute models arrived at consistent conclusions for those cases [40]. Building from those important accomplishments, the present work investigates the theory for adding attractive inter-atomic forces to those primitive cases.

The counter-intuitive ingredients of the statistical mechanical theory [36] together with apparent disagreement with some experiments [37, 41,43] that do involve attractive forces, lead promptly to questions about the consequences of solute attractive forces associated with simple hydrophobic solutes [4]. That issue has been broadly discussed several times over the intervening years [42, 4448] without achieving a definitive solution. That situation can now change on the basis of the new results for hydrophobic interactions noted above.

Distinctions [49] deriving from inclusion of solute attractive forces are exemplified in FIG. 1 and FIG. 2, Inclusion of solute attractive forces diminishes the strength of hydrophobic bonding: solvent attraction to the solute tends to pull the solute species apart. This behavior could be expected from sensitive appreciation [44, 48. of preceding results. The local molecular field theory (LMF) discussed below is a simple, persuasive theory for these effects of attractive interactions [50. Clarifying and testing that theory is the goal of this work.

Though the substantially the same theory we test below was known and used [44, 51, 52] long ago, the insight underlying recent discussions of LMF theory, e.g., [50, have considerably strengthened it. We here give a concise derivation with a clear analogy to a thermodynamic van der Waals picture, and is therefore unusually compelling. In the next section we outline the LMF theory. Numerical results, and conclusions are identified in Sec. IV. Methods for the several computational steps are collected in Sec. III.

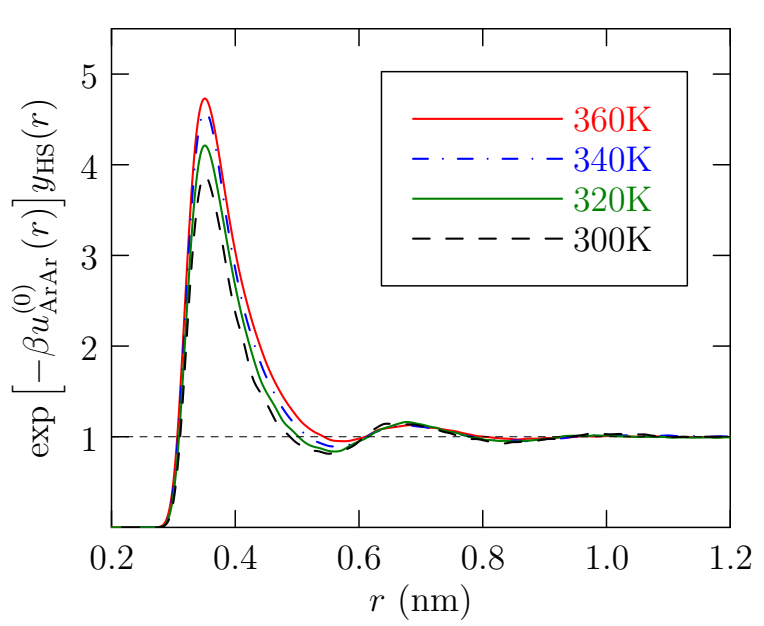

FIG. 1. Modeled radial distribution functions for WCA repulsive-force Ar solutes, based on the hard-sphere cavity distribution functions 39 .

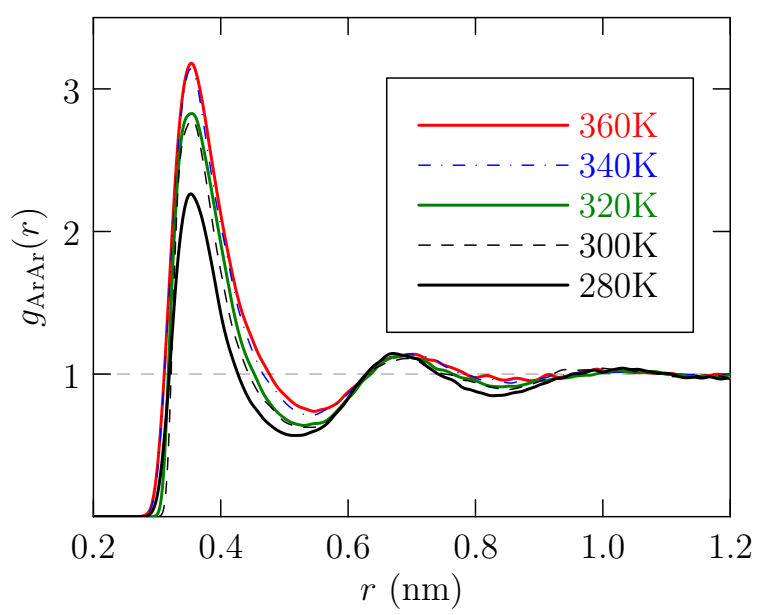

FIG. 2. Ar-Ar radial distribution functions reconstructed from stratified (window) calculations. Notice (compare FIG. 1) that contact hydrophobic interactions are weaker when solute attractive forces are included. In contrast, solvent-separated correlations are more strongly structured with inclusion of atomic attractive forces.

\section{LOCAL MOLECULAR FIELD THEORY}

The LMF idea is to study the inhomogeneous density of a fluid subject to an external field. We focus on the density structure resulting from the placement of an Ar atom at a specific location. That distinguished atom exerts an external field on the surrounding fluid and distorts the density. With $U$ the intermolecular potential energy function for the system and $\Phi$ the external field exerted by the distinguished atom, the resulting distorted density is $\rho_{\alpha \mathrm{M}}(\boldsymbol{r} ; U, \Phi)$ at position $\boldsymbol{r}$ of $\alpha$ atoms of a molecule of type M. The goal of the LMF theory is to analyze $\rho_{\alpha \mathrm{M}}(\boldsymbol{r} ; U, \Phi)$ on the basis of the characteristics of the interactions $U$ and $\Phi$.

We assume that a reference potential energy, de- 
noted by $U^{(0)}$, has been identified to help in analyzing $\rho_{\alpha \mathrm{M}}(\boldsymbol{r} ; U, \Phi)$. Specifically, our goal is the match

$$
\rho_{\alpha \mathrm{M}}(\boldsymbol{r} ; U, \Phi)=\rho_{\alpha \mathrm{M}}\left(\boldsymbol{r} ; U^{(0)}, \Phi^{(0)}\right),
$$

achieved for the reference system with interactions $U^{(0)}$, and an effective external field $\Phi^{(0)}$. That effective field is the objective of the analysis below. A successful match Eq. (1) establishes aspects of $U$ that can be treated as molecular mean-fields, thus offering a molecular mechanism for $\rho_{\alpha \mathrm{M}}(\boldsymbol{r} ; U, \Phi)$.

Identification of a reference potential energy function $U^{(0)}$ thus requires physical insight. One suggestion for the inter-atomic force fields of current simulation calculations corresponds to Gaussian-truncated electrostatic interactions associated with the partial charges of simulation models 50. In that case, the crucial difference

$$
U-U^{(0)}=\sum_{\alpha \mathrm{M}, \gamma \mathrm{M}^{\prime}} u_{\alpha \mathrm{M} \gamma \mathrm{M}^{\prime}}^{(1)}\left(\left|\boldsymbol{r}_{\alpha \mathrm{M}}-\boldsymbol{r}_{\gamma \mathrm{M}^{\prime}}\right|\right),
$$

is atom-pair decomposable. For the case of interest here, $u_{\alpha \mathrm{M} \gamma \mathrm{M}^{\prime}}^{(1)}\left(\left|\boldsymbol{r}_{\alpha \mathrm{M}}-\boldsymbol{r}_{\gamma \mathrm{M}^{\prime}}\right|\right)$ is the WCA-attractive part of the Lennard-Jones interactions associated with the Ar atoms [51].

In seeking the match Eq. (1), we adopt an atom-based perspective, and focus on the chemical potential [53],

$$
\begin{aligned}
\mu_{\alpha \mathrm{M}}=\beta^{-1} \ln \left[\rho_{\alpha \mathrm{M}}\right. & (\boldsymbol{r} ; U, \Phi) \Lambda_{\left.\alpha \mathrm{M}^{3}\right]} \\
& +\varphi_{\alpha \mathrm{M}}(\boldsymbol{r})+\mu_{\alpha \mathrm{M}}^{(\mathrm{ex})}(\boldsymbol{r} ; \rho, \beta U),
\end{aligned}
$$

of $\alpha \mathrm{M}$ atoms, which decomposes

$$
\Phi=\sum_{\alpha \mathrm{M}} \varphi_{\alpha \mathrm{M}}\left(\boldsymbol{r}_{\alpha \mathrm{M}}\right)
$$

Here the temperature is $T=\left(k_{\mathrm{B}} \beta\right)^{-1}$; the thermal deBroglie wavelength $\Lambda_{\alpha \mathrm{M}}$ depends only on $T$ and on fundamental parameters associated with $\alpha$ atoms. As indicated, the excess contribution $\mu_{\alpha \mathrm{M}}^{(\mathrm{ex})}(\boldsymbol{r} ; \rho, \beta U)$ depends functionally on $(\rho, \beta U)$, not on the external field.

For some simulation models, the atom-based $\mu_{\alpha \mathrm{M}}$ (Eq. (3) ) may raise questions regarding the operational status of atom chemical potentials. But this perspective would be satisfactory for ab initio descriptions of the solution, and is sufficiently basic that we do not further side-track this discussion. Similarly for the reference case

$$
\begin{aligned}
\mu_{\alpha \mathrm{M}}^{(0)}=\beta^{-1} \ln & {\left[\rho_{\alpha \mathrm{M}}^{(0)}\left(\boldsymbol{r} ; U^{(0)}, \Phi^{(0)}\right) \Lambda_{\alpha \mathrm{M}}{ }^{3}\right] } \\
& +\varphi_{\alpha \mathrm{M}}^{(0)}(\boldsymbol{r})+\mu_{\alpha \mathrm{M}}^{(\mathrm{ex})}\left(\boldsymbol{r} ; \rho^{(0)}, \beta U^{(0)}\right),
\end{aligned}
$$

with

$$
\Phi^{(0)}=\sum_{j} \varphi_{\alpha \mathrm{M}}^{(0)}\left(\boldsymbol{r}_{j}\right)
$$

The forms Eqs. (3) and (5) allows us to express the match Eq. (1) as

$$
\begin{aligned}
\varphi_{\alpha \mathrm{M}}^{(0)}(\boldsymbol{r}) & =\varphi_{\alpha \mathrm{M}}(\boldsymbol{r}) \\
+ & {\left[\mu_{\alpha \mathrm{M}}^{(\mathrm{ex})}(\boldsymbol{r} ; \rho, \beta U)-\mu_{\alpha \mathrm{M}}^{(\mathrm{ex})}\left(\boldsymbol{r} ; \rho, \beta U^{(0)}\right)\right] } \\
& + \text { constant } .
\end{aligned}
$$

The bracketed terms in Eq. (7) depend functionally on the densities, not on the external field. The constant in Eq. (7) involves the chemical potentials of the two systems.

The approximation

$$
\begin{aligned}
& \mu_{\alpha \mathrm{M}}^{(\mathrm{ex})}(\boldsymbol{r} ; \rho, \beta U) \approx \mu_{\alpha \mathrm{M}}^{(\mathrm{ex})}\left(\boldsymbol{r} ; \rho, \beta U^{(0)}\right) \\
& \quad+\sum_{\gamma \mathrm{M}^{\prime}} \int \rho_{\gamma \mathrm{M}^{\prime}}\left(\boldsymbol{r}^{\prime}\right) u_{\gamma \mathrm{M}^{\prime} \alpha \mathrm{M}}^{(1)}\left(\left|\boldsymbol{r}^{\prime}-\boldsymbol{r}\right|\right) \mathrm{d} \boldsymbol{r}^{\prime}
\end{aligned}
$$

is then transparently analogous to van der Waals theory [54 for the inclusion of attractive interactions, i.e., $\Delta \mu \approx$ $-2 a \rho$, with $a$ the van der Waals parameter describing attractive intermolecular interactions. Transcribing to the case of $\operatorname{Ar}(\mathrm{aq})$ at infinite dilution produces

$$
\begin{aligned}
\varphi_{\mathrm{Ar}}^{(0)}(\boldsymbol{r}) \approx & \varphi_{\mathrm{Ar}}(\boldsymbol{r}) \\
& +\int\left[\rho_{\mathrm{O}}\left(\boldsymbol{r}^{\prime}\right)-\rho_{\mathrm{O}}\right] u_{\mathrm{OAr}}^{(1)}\left(\left|\boldsymbol{r}^{\prime}-\boldsymbol{r}\right|\right) \mathrm{d} \boldsymbol{r}^{\prime} .
\end{aligned}
$$

The fields vanish far from their source, and therefore the constant contribution of Eq. (7) is accommodated explicitly in Eq. (9). This argument matches the results of Rodgers and Weeks [55] in the several instances they considered. Derivations that emphasize alternative (electrostatic) interactions are available elsewhere [56 58].

Though the statistical mechanical theory of Eq. 9 is simple, the field sought depends on the density, which depends on the field. A linear statistical mechanical approximation Eq. (8) produces the non-linear Eq. (9) to solve. The non-linearity is not an obstacle here because the densities on the right of Eq. (9) are straightforwardly obtained from routine simulation (FIG. 3, see also [59]). Notice (FIG. 3) that the effects of attractive ArO interactions on ArO correlations are modest, as was argued long ago 44].

Now consider $\rho_{\mathrm{Ar}}\left(\boldsymbol{r} ; U^{(0)}, \Phi^{(0)}\right)$, the density of $\mathrm{Ar}$ atoms without attractive interactions $\beta u_{\mathrm{OAr}}^{(1)}(\boldsymbol{r})$ but experiencing the effective field $\beta \varphi_{\mathrm{Ar}}^{(0)}(\boldsymbol{r})$. We approximate 62

$$
\begin{gathered}
\ln \rho_{\mathrm{Ar}}\left(\boldsymbol{r} ; U^{(0)}, \Phi^{(0)}\right) / \rho_{\mathrm{Ar}} \approx-\beta \varphi_{\mathrm{Ar}}^{(0)}(\boldsymbol{r})+\ln y_{\mathrm{HS}}(\boldsymbol{r}) \\
=-\beta\left(\varphi_{\mathrm{Ar}}^{(0)}(\boldsymbol{r})-u_{\mathrm{ArAr}}^{(0)}(\boldsymbol{r})\right)+\ln g_{\mathrm{ArAr}}^{(0)}(\boldsymbol{r})
\end{gathered}
$$

adopting the repulsive-force solute results of FIG. 11. The field $\varphi_{\mathrm{Ar}}^{(0)}(\boldsymbol{r})$ incorporates aspects of the intermolecular 


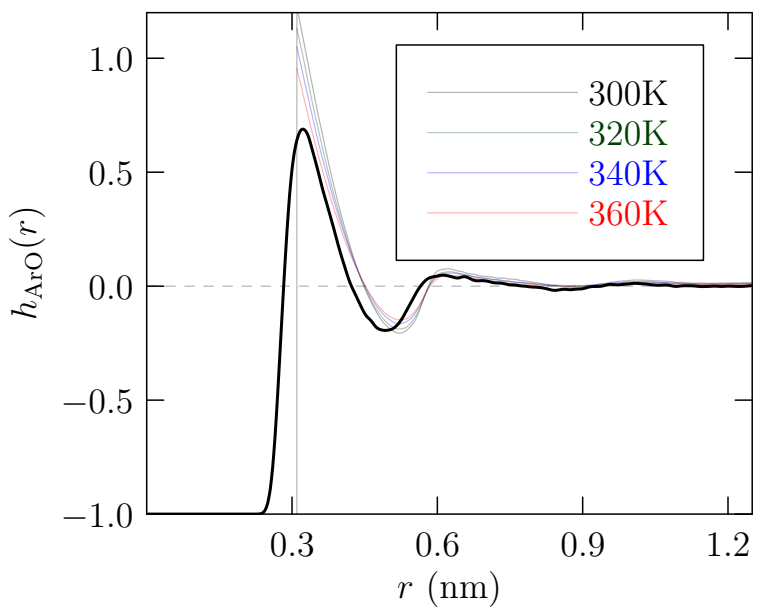

FIG. 3. Observed radial correlation of $\mathrm{O}$ atoms with an Ar atom, $T=300 \mathrm{~K}, p=1 \mathrm{~atm}$ (heavy curve). Correlation functions (fainter, background curves) for hard-sphere model solutes with distances of closest approach $0.31 \mathrm{~nm}$ (FIG. 1) on the basis of cavity methods 39, 59, from Chaudhari 49. The PC theory 35 predictions for the maxima of the hard sphere correlation functions would be close to 2, larger than these numerical results 35, 60. For the soft-sphere case, attractive van der Waals interactions draw-in near-neighbor O-atoms slightly 39, 61. Since attractions draw-in, rather than drawup, the attractive interactions case is not wetter than the reference case.

attractions as mean-field effects according to Eq. (9). The match Eq. (1) pairs this with

$$
\ln \rho_{\mathrm{Ar}}(\boldsymbol{r} ; U, \Phi) / \rho_{\mathrm{Ar}}=\ln g_{\mathrm{ArAr}}(\boldsymbol{r}) .
$$

Combining with Eq. 10

$$
\begin{aligned}
& \ln g_{\mathrm{ArAr}}(\boldsymbol{r})=\ln g_{\mathrm{ArAr}}^{(0)}(\boldsymbol{r})-\beta\left(\varphi_{\mathrm{Ar}}(\boldsymbol{r})-u_{\mathrm{ArAr}}^{(0)}(\boldsymbol{r})\right) \\
&-\int\left[\rho_{\mathrm{O}}\left(\boldsymbol{r}^{\prime}\right)-\rho_{\mathrm{O}}\right] \beta u_{\mathrm{OAr}}^{(1)}\left(\left|\boldsymbol{r}^{\prime}-\boldsymbol{r}\right|\right) \mathrm{d} \boldsymbol{r}^{\prime} .
\end{aligned}
$$

Finally noting $\varphi_{\mathrm{Ar}}=u_{\mathrm{ArAr}}^{(0)}+u_{\mathrm{ArAr}}^{(1)}$ and rearranging yields

$$
\begin{aligned}
-\ln \left[\frac{g_{\mathrm{ArAr}}(\boldsymbol{r})}{g_{\mathrm{ArAr}}^{(0)}(\boldsymbol{r})}\right] & \approx \beta u_{\mathrm{ArAr}}^{(1)}(\boldsymbol{r}) \\
& +\int h_{\mathrm{ArO}}\left(\boldsymbol{r}^{\prime}\right) \rho_{\mathrm{O}} \beta u_{\mathrm{OAr}}^{(1)}\left(\left|\boldsymbol{r}^{\prime}-\boldsymbol{r}\right|\right) \mathrm{d} \boldsymbol{r}^{\prime}
\end{aligned}
$$

\section{A. Comparison to EXP theory [44]}

As noted above, the approximate theory Eq. (13) requires $h_{\mathrm{ArO}}(\boldsymbol{r})$, and we can conveniently take that from routine simulation. The corresponding approximate the- ory for $h_{\mathrm{ArO}}(\boldsymbol{r})$ is

$$
\begin{aligned}
-\ln \left[\frac{g_{\mathrm{ArO}}(\boldsymbol{r})}{g_{\mathrm{ArO}}^{(0)}(\boldsymbol{r})}\right] & \approx \beta u_{\mathrm{OAr}}^{(1)}(\boldsymbol{r}) \\
& +\int h_{\mathrm{OO}}\left(\boldsymbol{r}^{\prime}\right) \rho_{\mathrm{O}} \beta u_{\mathrm{OAr}}^{(1)}\left(\left|\boldsymbol{r}^{\prime}-\boldsymbol{r}\right|\right) \mathrm{d} \boldsymbol{r}^{\prime},
\end{aligned}
$$

where $h_{\mathrm{OO}}(\boldsymbol{r})$ is the observed $\mathrm{OO}$ correlation function for pure water. Acknowledging closure approximations specific to traditional implementations, this is just the EXP approximation 51 applied to this correlation problem long ago [44, 52]. This observation serves further to identify Eq. (13) as a relative of the EXP approximation also. Nevertheless, the distinction between the theory of Ref. 44, with its specific implementation details, from Eq. (13) should be kept in mind. The most prominent distinction is that Eq. (13) exploits $h_{\mathrm{ArO}}(\boldsymbol{r})$ evaluated self-consistently or, here, the numerically exact result.

Note further that the Eq. (13) offers additional possibilities compared to Eq. (14) for variety of outcomes because of possibilities from imbalance of $u_{\mathrm{OAr}}^{(1)}(\boldsymbol{r})$ and $u_{\mathrm{ArAr}}^{(1)}(\boldsymbol{r})$.

\section{B. Perspective from quasi-chemical theory} [44, 48, 63]

Quasi-chemical theory (QCT) provides insight into the LMF approximation Eq. (13). Since QCT is designed to evaluate interaction contributions to chemical potentials, 39, 61, 64 Eq. (7) is the relevant starting point. From the QCT formulation [48, 63, the packing contributions to those two chemical potentials are identical, and cancel each other. Next to be considered [48] is the mean hydration energy, denoted by $\left\langle\varepsilon \mid r, n_{\lambda}=0\right\rangle$, of the Ar appearing at $r$. That previous QCT effort 48] observed that the outer-shell QCT fluctuation contribution was comparatively small. Thus $\left\langle\varepsilon \mid r, n_{\lambda}=0\right\rangle$ is the leading factor in describing the effect of attractive interactions being added [44, 48. In the QCT study, 48] $\left\langle\varepsilon \mid r, n_{\lambda}=0\right\rangle$ was evaluated from molecular simulation data. The PC modeling of long-ago [44 recognized the importance of $\left\langle\varepsilon \mid r, n_{\lambda}=0\right\rangle$, and used a RISM approximation to incorporate the specific structure of the $\mathrm{Ar}_{2}$ diatom. Returning to the LMF theory, the right-most of Eq. (8) addresses $\left\langle\varepsilon \mid r, n_{\lambda}=0\right\rangle$, but does not calculate it for the detailed $\mathrm{Ar}_{2}$ geometry. Incomplete evaluation of $\left\langle\varepsilon \mid r, n_{\lambda}=0\right\rangle$ is thus the chief neglect of the present LMF theory.

\section{METHODS}

\section{A. Simulations}

The simulations were carried-out with the GROMACS package [65, the SPC/E model of the water molecules 


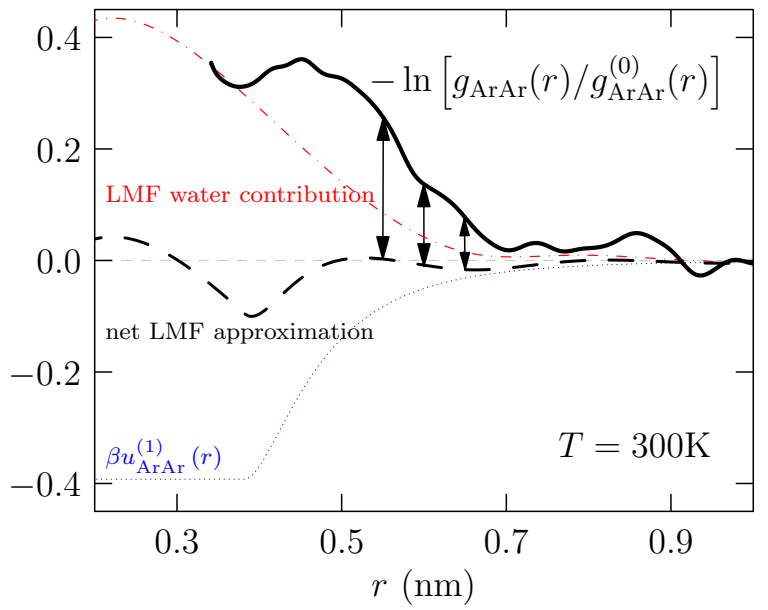

FIG. 4. Test of the LMF theory, Eq 13. The net result for the LMF approximation (black, dashed, right-side of Eq. 13p) is the sum of the direct interaction (blue, dotted curve) and the water contribution (red, dotdashed curve, Eq. (17)). $-\ln \left[g_{\mathrm{ArAr}}(r) / g_{\mathrm{ArAr}}^{(0)}(r)\right]$ differs from the net LMF approximation both in contact and solvent-separated configurations.

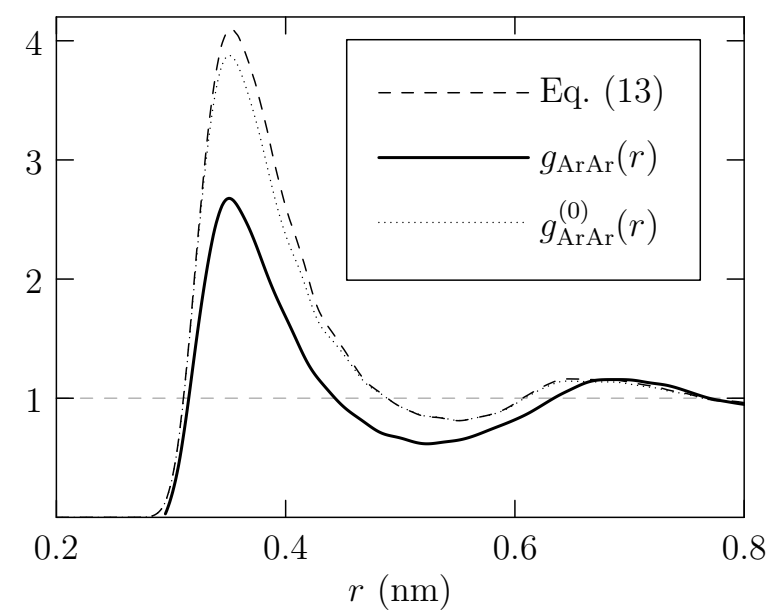

FIG. 5. Comparison of LMF approximation Eq. 13 with $g_{\mathrm{ArAr}}^{(0)}(r)$ (reference system, fainter, dotted curve) and $g_{\mathrm{ArAr}}(r)$. Note the significantly different behavior of $g_{\mathrm{ArAr}}^{(0)}(r)$ and $g_{\mathrm{ArAr}}(r)$ in the second shell, not addressed by this approximation.

[66, and the OPLS force field. GROMACS selects SETTLE 67] constraint algorithm for rigid SPC/E water molecules. The same constraint algorithm was used in previous simulations involving water 39,68 . Standard periodic boundary conditions were employed, with particle mesh Ewald utilizing a cutoff of $1 \mathrm{~nm}$ and long-range dispersion corrections applied to energy and pressure. The Parrinello-Rahman barostat controlled the pressure at $1 \mathrm{~atm}$, and the Nose-Hoover thermostat was used to maintain the temperature. The simulation cell for the $\operatorname{Ar}(\mathrm{aq})$ system consisted of two (2) argon molecules and
1000 water molecules. Initial configurations were constructed with PACKMOL [69] to construct a system close to the density of interest. The solute-solute separation spanning $0.33 \mathrm{~nm}$ to $1.23 \mathrm{~nm}$ was stratified using a standard windowing approach and the results combined using the weighted histogram analysis method (WHAM) [70]. This involved 19 windows (and simulations) for window separations $r$ ranging from $0.33 \mathrm{~nm}$ to $1.23 \mathrm{~nm}$.

\section{B. Implementation of LMF theory}

With the information of FIG. 3, the LMF approximation Eq. 13 depends only linearly on the attractive interactions. We evaluated Eq. (13) standardly, introducing the spatial Fourier transforms

$$
\hat{u}_{\mathrm{OAr}}^{(1)}(k)=\int u_{\mathrm{OAr}}^{(1)}(r)\left(\frac{\sin k r}{k r}\right) \mathrm{d} \boldsymbol{r},
$$

and

$$
\hat{h}_{\mathrm{OAr}}(k)=\int h_{\mathrm{OAr}}(r)\left(\frac{\sin k r}{k r}\right) \mathrm{d} \boldsymbol{r} .
$$

Then

$$
\begin{aligned}
\int \hat{h}_{\mathrm{ArO}}(k) \rho_{\mathrm{O}} \beta \hat{u}_{\mathrm{OAr}}^{(1)}(k)\left(\frac{\sin k r}{k r}\right) \frac{\mathrm{d} \boldsymbol{k}}{(2 \pi)^{3}} \\
=\int h_{\mathrm{ArO}}\left(\boldsymbol{r}^{\prime}\right) \rho_{\mathrm{O}} \beta u_{\mathrm{OAr}}^{(1)}\left(\left|\boldsymbol{r}^{\prime}-\boldsymbol{r}\right|\right) \mathrm{d} \boldsymbol{r}^{\prime} .
\end{aligned}
$$

The parameters for this application are $\rho_{\mathrm{O}}=33.8 / \mathrm{nm}^{3}$, $\varepsilon_{\mathrm{OAr}}=0.798 \mathrm{~kJ} / \mathrm{mol}, \sigma_{\mathrm{OAr}}=0.328 \mathrm{~nm}, \varepsilon_{\mathrm{ArAr}}=$ $0.978 \mathrm{~kJ} / \mathrm{mol}$, and $\sigma_{\mathrm{ArAr}}=0.340 \mathrm{~nm}$.

\section{Osmotic $B_{2}$ and Infinite Size Extrapolation}

The distribution function $g_{\mathrm{ArAr}}(r)=h_{\mathrm{ArAr}}(r)+1$ provides access to the osmotic second virial coefficient,

$$
B_{2}=-\frac{1}{2} \lim _{\rho_{\mathrm{Ar}} \rightarrow 0} \int h_{\mathrm{ArAr}}(r) \mathrm{d}^{3} r .
$$

We utilize the extrapolation procedure of Krüger, et al. [71, 72

$$
-2 B_{2}=\lim _{R \rightarrow \infty} 4 \pi \int_{0}^{2 R} h_{\mathrm{ArAr}}(r) w(r / 2 R) r^{2} \mathrm{~d} r,
$$

with

$$
w(x)=1-\left(\frac{3}{2}\right) x+\left(\frac{1}{2}\right) x^{3} .
$$

Computed values for $1 / 2 R>0$ were least-squares fitted with a polynomial quadratic order in $1 / 2 R$, then extrapolated to $1 / 2 R=0$. This procedure has been successfully tested [68, 73, 74] and does not require further statistical mechanical theory. 


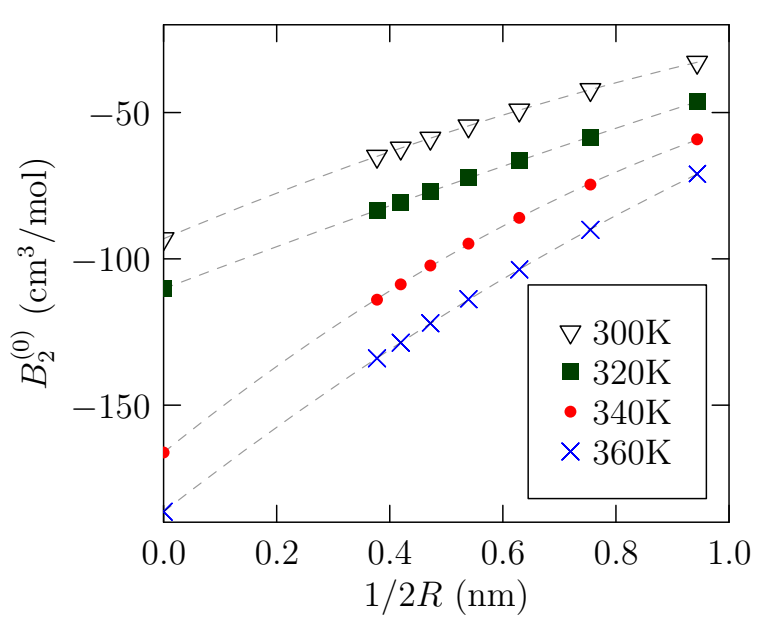

FIG. 6. Extrapolation to evaluate the osmotic second virial coefficient, $B_{2}^{(0)}$, for the WCA repulsive-force Ar solutes (FIG. 1). The symbol at $1 / 2 R=0$ is the extrapolated value; see Sec III C Hydrophobic interactions gauged by $B_{2}^{(0)}$ become more attractive with increasing temperature in this range.

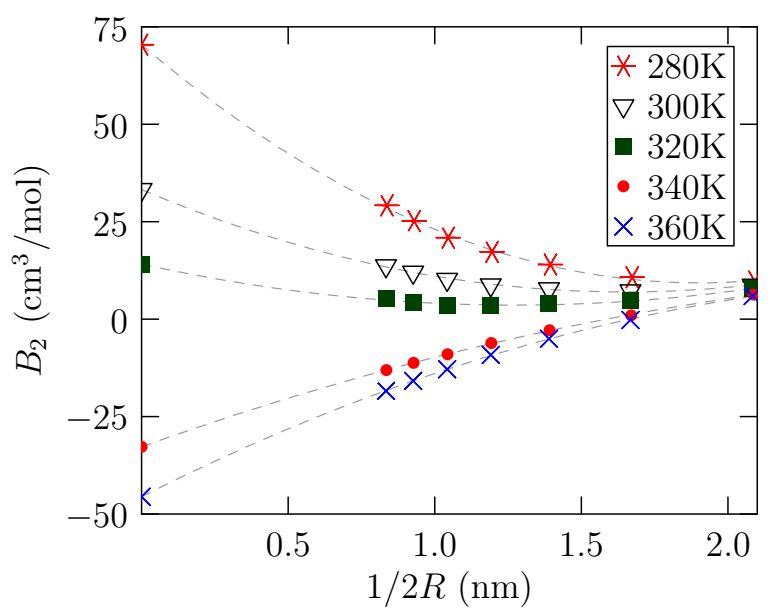

FIG. 7. Extrapolation (Sec IIIC) to evaluate the osmotic second virial coefficient, $B_{2}$, for the full attractions case of FIG. 2. Comparing with FIG. 6, we see that inclusion of solute attractive-forces makes these $B_{2}$ more positive (repulsive). Hydrophobic interactions gauged by $B_{2}$ become more attractive with increasing temperature in this range. $B_{2}$ changes from positive to negative values in $T=(320 \mathrm{~K}, 340 \mathrm{~K})$. Thus $B_{2} \approx 0$ in that interval, qualitatively consistent with the work of Watanabe, et al., 45].

\section{RESULTS AND DISCUSSION}

Changing purely repulsive atomic interactions to include realistic attractions diminishes the strength of hydrophobic bonds (FIGs. 1 and 2). Within this LMF theory, and also the earliest theories for this [44, 48, the hydration environment competes with direct Ar-Ar attractive interactions (FIG. 4). The outcome of that competition is sensitive to the differing strengths of the at- tractive interactions. The earlier application [44] used the EXP approximation to analyze the available Monte Carlo calculations on atomic LJ solutes in water [75]. That theoretical modelling found modest effects of attractive interactions, and encouraging comparison with the Monte Carlo results. This application of the LMF theory (Eq. 13) again predicts modest effects of attractive interactions, but the net comparison from the simulation results shows big differences. The outcome alternative to the historical work is due to the fact that the earlier theory used the PC approximate results for the reference system $g_{\mathrm{ArAr}}^{(0)}(r)$, and we now know that approximation is not accurate for this application [39, despite being the only theory available. Here the LMF theory (Eq. 13) predicts modest-sized changes, though in addition it predicts changes opposite in sign to the observed changes. Note further that $g_{\mathrm{ArAr}}^{(0)}(r)$ and $g_{\mathrm{ArAr}}(r)$ differ distinctively in the second hydration shell, and those differences suggest more basic structural changes driven by attractive interactions.

The earliest study of these effects [44] went further to analyze a Lennard-Jones model of $\mathrm{CH}_{4}-\mathrm{CH}_{4}$ (aq) with much larger attractive interactions. The theory developed for that application was successful for the case studied [47, but the correspondence of that LJ model to $\mathrm{CH}_{4}$ solutes was not accurate enough [47. to warrant further interest.

That earlier theory featured study of $\left\langle\varepsilon \mid r, n_{\lambda}=0\right\rangle$ that has acquired a central role in QCT study of the present problem [48. A more accurate evaluation would involve $n$-body $(n>2)$ correlations, perhaps even treated by natural superposition approximations [76. Detailed treatment of the $\mathrm{Ar}_{2}$ diatom geometry is the most prominent difference between that QCT approach and the present LMF theory (Eq. (13)). Nevertheless, a full QCT analysis of these differences is clearly warranted and should be the subject of subsequent study.

These changes due to attractive interatomic interactions are directly reflected in the values of $B_{2}$ (FIGs. 6 and 77. Slight curvature of the extrapolation (FIGs. 6 and 77) is evident but, in view of the previous testing 68, 73, 74, not concerning. In all cases here, $B_{2}$ becomes more attractive with increasing temperature below $T=$ $360 \mathrm{~K}$. This behavior is consistent with accumulated experience and recently obtained results $[39,40,73$. With attractive interactions in play, $B_{2}$ can change from positive to negative values with increasing temperatures. This is consistent with the historical work of Watanabe, et al., [45] that $B_{2} \approx 0$ for intermediate cases.

Finally, we emphasize that since attractions make substantial contributions, precise tests of the PC theory [2, 75] with results on cases with realistic attractive interactions should specifically address the role of attractive interactions that were not included in the PC theory. 


\section{ACKNOWLEDGEMENT}

We thank J. D. Weeks (University of Maryland) for helpful discussions. Sandia is a multiprogram laboratory operated by Sandia Corporation, a Lockheed Martin Company, for the U.S. Department of Energy's Na- tional Nuclear Security Administration under Contract No. DE-AC04-94AL8500. The financial support of Sandia's LDRD program and the Gulf of Mexico Research Initiative (Consortium for Ocean Leadership Grant SA 12-05/GoMRI-002) is gratefully acknowledged.
[1] Kauzmann, W. (1987) Thermodynamics of unfolding. Nature 325, 763-764.

[2] Garde, S., Hummer, G., García, A. E., Paulaitis, M. E., and Pratt, L. R. (1996) Origin of entropy convergence in hydrophobic hydration and protein folding. Phys. Rev. Letts. 77, $4966-4968$.

[3] Tanford, C. (1997) How Protein Chemists Learned about the Hydrophobic Factor. Protein Science 6, 1358-1366.

[4] Silverstein, T. P. (2008) Hydrophobic Solvation NOT via Clathrate Water Cages. J. Chem. Ed. 85, 917-918.

[5] Murayama, M. (1966) Molecular Mechanism of Red Cell "Sickling". Science 153, 145-149.

[6] Urry, D. W., Kuan, C. L., Parker, T. M., Gowda, D. C., Prasad, K. U., Reid, M. C., and Safavy, A. (1991) Temperature of polypeptide inverse temperature transition depends on mean residue hydrophobicity. J. Am. Chem. Soc 113, 4346-4348.

[7] Urry, D. W. (1982) Characterization of soluble peptides of elastin by physical techniques. Methods in Enzymology 82, 673-716.

[8] Nuhn, H., and Klok, H.-A. (2008) Secondary Structure Formation and LCST Behavior of Short Elastin-Like Peptides. Biomacromol. 9, 2755-2763.

[9] Reiersen, H., Clarke, A. R., and Rees, A. R. (1998) Short Elastin-like Peptides Exhibit the Same Temperatureinduced Structural Transitions as Elastin Polymers: Implications for Protein Engineering. J. Mol. Bio. 283, 255264.

[10] Nicolini, C., Ravindra, R., Ludolph, B., and Winter, R. (2004) Characterization of the Temperature- and Pressure-Induced Inverse and Reentrant Transition of the Minimum Elastin-Like Polypeptide GVG(VPGVG) by DSC, PPC, CD, and FT-IR Spectroscopy. Biophys. J. 86, 1385-1392.

[11] Li, B., Alonso, D. O. V., and Daggett, V. (2001) The molecular basis for the inverse temperature transition of elastin. J. Mol. Bio. 305, 581-592.

[12] Li, B., Alonso, D. O. V., Bennion, B. J., and Daggett, V. (2001) Hydrophobic Hydration Is an Important Source of Elasticity in Elastin-Based Biopoly. J. Am. Chem. Soc. 123, 11991-11998.

[13] Li, B., and Daggett, V. (2002) Molecular basis for the extensibility of elastin. J. Muscle Res. 83 Cell Mot. 23, $561-573$.

[14] Rousseau, R., Schreiner, E., Kohlmeyer, A., and Marx, D. (2004) Temperature-Dependent Conformational Transitions and Hydrogen-Bond Dynamics of the Elastin-Like Octapeptide GVG(VPGVG): A MolecularDynamics Study. Biophys. J. 86, 1393-1407.

[15] Schreiner, E., Nicolini, C., Ludolph, B., Ravindra, R., Otte, N., Kohlmeyer, A., Rousseau, R., Winter, R., and Marx, D. (2004) Folding and Unfolding of an Elastinlike Oligopeptide: "Inverse Temperature Transition," Reen- trance, and Hydrogen-Bond Dynamics. Phys. Rev. Letts. 92, 148101.

[16] Baer, M., Schreiner, E., Kohlmeyer, A., Rousseau, R., and Marx, D. (2006) Inverse Temperature Transition of a Biomimetic Elastin Model: Reactive Flux Analysis of Folding/Unfolding and Its Coupling to Solvent Dielectric Relaxation. J. Phys. Chem. B 110, 3576-3587.

[17] Krukau, A., Brovchenko, I., and Geiger, A. (2007) Temperature-Induced Conformational Transition of a Model Elastin-like Peptide GVG(VPGVG) 3 in Water. Biomacromol. 8, 2196-2202.

[18] Li, N. K., Quiroz, F. G., Hall, C. K., Chilkoti, A., and Yingling, Y. G. (2014) Molecular Description of the LCST Behavior of an Elastin-Like Polypeptide. Biomacromol. 15, 3522-3530.

[19] Maeda, Y., Higuchi, T., and Ikeda, I. (2000) Change in Hydration State during the Coil-Globule Transition of Aqueous Solutions of Poly(N-isopropylacrylamide) as Evidenced by FTIR Spectroscopy. Langmuir 16, 75037509.

[20] Qiu, X.-P., Tanaka, F., and Winnik, F. M. (2007) Temperature-induced phase transition of well-defined cyclic poly(N-isopropylacrylamide)s in aqueous solution. Macromol. 40, 7069-7071.

[21] Bae, Y. C., Shim, D. S., J. J .and Soane, and Prausnitz, J. M. (1993) Representation of vapor-liquid and liquid-liquid equilibria for binary systems containing polymers: Applicability of an extended Flory-Huggins equation. J. Appl. Poly. Sci. 47, 1193-1206.

[22] Hummer, G., Garde, S., García, A. E., Pohorille, A., and Pratt, L. R. (1996) An Information Theory Model of Hydrophobic Interactions. Proc. Natl. Acad. Sci. USA 93, 8951-8955.

[23] Gomez, M. A., Pratt, L. R., Hummer, G., and Garde, S. (1999) Molecular Realism in Default Models for Information Theories of Hydrophobic Effects. J. Phys. Chem. B 103, 3520-3523.

[24] Ashbaugh, H., and Pratt, L. (2006) Colloquium: scaled particle theory and the length scales of hydrophobicity. Rev. Mod. Phys. 78, 159-178.

[25] Ashbaugh, H. S., and Pratt, L. R. (2007) Contrasting Nonaqueous against Aqueous Solvation on the Basis of Scaled-Particle Theory. J. Phys. Chem. B 111, 93309336.

[26] Pratt, L. (2002) Molecular theory of hydrophobic effects: "She is too mean to have her name repeated". Ann. Rev. Phys. Chem. 53, 409-436.

[27] Pratt, L. R., Garde, S., and Hummer, G. New Approaches to Problems in Liquid State Theory; Springer, Netherlands, 1999; pp 407-420.

[28] Hummer, G., Garde, S., García, A. E., Paulaitis, M. E., and Pratt, L. R. (1998) Hydrophobic Effects on a Molecular Scale. J. Phys. Chem. B 102, 10469-10482. 
[29] Pratt, L. R., and Pohorille, A. (2002) Hydrophobic effects and modeling of biophysical aqueous solution interfaces. Chem. Rev. 102, 2671-2692.

[30] Pierotti, R. A. (1967) On the scaled-particle theory of dilute aqueous solutions. J. Phys. Chem 71, 2366-2367.

[31] Pierotti, R. A. (1976) A scaled particle theory of aqueous and nonaqueous solutions. Chem. Rev. 76, 717-726.

[32] Pierotti, R. A. (1965) Aqueous Solutions of Nonpolar Gases. J. Phys. Chem 69, 281-288.

[33] Ben-Naim, A., and Friedman, H. L. (1967) On the application of the scaled particle theory to aqueous solutions of nonpolar gases. J. Phys. Chem 71, 48-49.

[34] Stillinger, F. H. (1973) Structure in aqueous solutions of nonpolar solutes from the standpoint of scaled-particle theory. J. Soln. Chem. 2, 141.

[35] Pratt, L. R., and Chandler, D. (1977) Theory of the hydrophobic effect. J. Chem. Phys. 67, 3683-3704.

[36] Chan, D. Y. C., Mitchell, D. J., Ninham, B. W., and Pailthorpe, B. A. In Recent Advances; Franks, F., Ed.; Water: A Comprehensive Treatise; Plenum: New York, 1979; Vol. 6; pp 239-278.

[37] Rossky, P. J., and Friedman, H. L. (1980) Benzenebenzene interaction in aqueous solution. J. Phys. Chem. $84,587-589$.

[38] Pratt, L. R. (1985) Theory of hydrophobic effects. Ann. Rev. Phys. Chem. 36, $433-449$.

[39] Chaudhari, M. I., Holleran, S. A., Ashbaugh, H. S., and Pratt, L. R. (2013) Molecular-scale hydrophobic interactions between hard-sphere reference solutes are attractive and endothermic. Proc. Nat. Acad. Sci. USA 110, 2055720562.

[40] Koga, K. (2013) Osmotic second virial coefficient of methane in water. J. Phys. Chem. B 117, 12619-12624.

[41] Tucker, E. E., and Christian, S. D. (1979) A prototype hydrophobic interaction. The dimerization of benzene in water. J. Phys. Chem. 83, 426-427.

[42] Pratt, L. R. (1985) Theory of Hydrophobic Effects. Ann. Rev. Phys. Chem. 36, 433-449.

[43] Bernal, P. J., Christian, S. D., and Tucker, E. E. (1986) Vapor-pressure studies of hydrophobic association - thermodynamics of fluorobenzene in dilute aqueous-solution. J. Soln. Chem. 15, 947-956.

[44] Pratt, L. R., and Chandler, D. (1980) Effects of solutesolvent attractive forces on hydrophobic correlations. $J$. Chem. Phys. 73, $3434-41$.

[45] Watanabe, K., and Andersen, H. C. (1986) Moleculardynamics study of the hydrophobic interaction in an aqueous-solution of krypton. J. Phys. Chem. 90, $795-$ 802.

[46] Smith, D. E., Zhang, L., and Haymet, A. D. J. (1992) Entropy of association of methane in water: a new molecular dynamics computer simulation. J. Am. Chem. Soc. 114, 5875-5876.

[47] Smith, D. E., and Haymet, A. D. J. (1993) Free energy, entropy, and internal energy of hydrophobic interactions: computer simulations. J. Chem. Phys. 98, 6445-6454.

[48] Asthagiri, D., Merchant, S., and Pratt, L. R. (2008) Role of attractive methane-water interactions in the potential of mean force between methane molecules in water. $J$. Chem. Phys. 128, 244512.

[49] Chaudhari, M. I. Molecular Simulations to Study Thermodynamics of Polyethylene Oxide Solutions. Ph.D. thesis, Department of Chemical \& Biomolecular Engineering, Tulane University, 2013.
[50] Rodgers, J. M., and Weeks, J. D. (2008) Local molecular field theory for the treatment of electrostatics. J. Phys.: Condens. Matter 20, 494206.

[51] Hansen, J.-P., and McDonald, I. R. Theory of Simple Liquids; Academic Press, 1976.

[52] Hummer, G., and Garde, S. (1998) Cavity expulsion and weak dewetting of hydrophobic solutes in water. Phys. Rev. Lett. 80, 4193-4196.

[53] Beck, T. L., Paulaitis, M. E., and Pratt, L. R. The Potential Distribution Theorem and Models of Molecular Solutions; Cambridge University Press, 2006.

[54] Widom, B. (1967) Intermolecular forces and the nature of the liquid state. Science 157, 375-382.

[55] Rodgers, J. M., and Weeks, J. D. (2008) Local molecular field theory for the treatment of electrostatics. J. Phys.: Condens. Matter 20.

[56] Rodgers, J. M., and Weeks, J. D. (2009) Accurate thermodynamics for short-ranged truncations of Coulomb interactions in site-site molecular models. J. Chem. Phys. 131, 244108.

[57] Rodgers, J. M., Hu, Z., and Weeks, J. D. (2011) On the efficient and accurate short-ranged simulations of uniform polar molecular liquids. Mol. Phys. 109, 1195-1211.

[58] Archer, A. J., and Evans, R. (2013) Relationship between local molecular field theory and density functional theory for non-uniform liquids. J. Chem. Phys. 138, 014502.

[59] Pratt, L. R., and Pohorille, A. In Proceedings of the EBSA 1992 International Workshop on WaterBiomolecule Interactions; Palma, M. U., PalmaVittorelli, M. B., and Parak, F., Eds.; Societá Italiana de Fisica: Bologna, 1993; pp 261-268.

[60] Ashbaugh, H. S., and Pratt, L. R. (2006) Colloquium: Scaled particle theory and the length scales of hydrophobicity. Rev. Mod. Phys. 78, 159-178.

[61] Asthagiri, D., Ashbaugh, H. S., Piryatinski, A., Paulaitis, M. E., and Pratt, L. R. (2007) Non-van der Waals treatment of the hydrophobic solubilities of $\mathrm{CF}_{4}$. J. Am. Chem. Soc. 129, $10133-10140$.

[62] Chandler, D., and Weeks, J. D. (1970) Equilibrium structure of simple liquids. Phys. Rev. Letts. 25, 149-152.

[63] Rogers, D. M., Jiao, D., Pratt, L. R., and Rempe, S. B. (2012) Structural Models and Molecular Thermodynamics of Hydration of Ions and Small Molecules. Ann. Rep. Comp. Chem. 8, 71-127.

[64] Sabo, D., Varma, S., Martin, M. G., and Rempe, S. B. (2008) Studies of the Thermodynamic Properties of Hydrogen Gas in Bulk Water. J. Phys. Chem. B 112, 867876 .

[65] Hess, B., Kutzner, C., van der Spoel, D., and Lindahl, E. (2008) GROMACS 4: Algorithms for highly efficient, load-balanced, and scalable molecular simulation. J. Chem. Theory. Comput. 4, 435-447.

[66] Berendsen, H. J. C., Grigera, J. R., and Straatsma, T. P. (1987) The missing term in effective pair potentials. $J$ Phys Chem 91, 6269-6271.

[67] Miyamoto, S., and Kollman, P. A. (1992) Settle: An analytical version of the SHAKE and RATTLE algorithm for rigid water models. J. Comp. Chem. 13, 952-962.

[68] Chaudhari, M. I., Sabo, D., Pratt, L. R., and Rempe, S. B. (2014) Hydration of $\mathrm{Kr}(\mathrm{aq})$ in dilute and concentrated solutions. J. Phys. Chem. B

[69] Martinez, L., Andrade, R., Birgin, E. G., and Martinez, J. M. (2009) PACKMOL: A package for building initial configurations for molecular dynamics simulations. 
J. Comp. Chem. 30, 2157-2164.

[70] Kumar, S., Rosenberg, J. M., Bouzida, D., Swedsen, R. H., and Kollman, P. A. (1992) The weighted histogram analysis method for free-energy calculation on biomolecules. I. The method. J. Comp. Chem. 13, 10111021.

[71] Krüger, P., Schnell, S. K., Bedeaux, D., Kjelstrup, S., Vlugt, T. J., and Simon, J.-M. (2012) Kirkwood-Buff integrals for finite volumes. J. Phys. Chem. Letts. 4, 235238.

[72] Schnell, S. K., Englebienne, P., Simon, J.-M., Krüger, P., Balaji, S. P., Kjelstrup, S., Bedeaux, D., Bardow, A., and Vlugt, T. J. (2013) How to apply the KirkwoodBuff theory to individual species in salt solutions. Chem. Phys. Letts. 582, 154-157.

[73] Ashbaugh, H. S., Weiss, K., Williams, S. M., Meng, B., and Surampudi, L. N. (2015) Temperature and Pressure
Dependence of Methane Correlations and Osmotic Second Virial Coefficients in Water. J. Phys. Chem. B 119, 6280-6294.

[74] Zhang, W. Multi-scale statistical theory and molecular simulation of electrolyte solutions. 2015; http://search. proquest. com/docview/1688677557?accountid=14437, Copyright - Copyright ProQuest, UMI Dissertations Publishing 2015.

[75] Pangali, C., Rao, M., and Berne, B. J. (1979) A Monte Carlo simulation of the hydrophobic interaction. J. Chem. Phys. 71, 2975-2981.

[76] Ashbaugh, H. S., Pratt, L. R., Paulaitis, M. E., Clohecy, J., and Beck, T. L. (2005) Deblurred Observation of the Molecular Structure of an Oil-Water Interface. J. Am. Chem. Soc 127, 2808-2809. 